\title{
Positive Psychologists on Positive Psychology: Ryan Niemiec
}

\author{
Interview by \\ Aaron Jarden
}

\begin{abstract}
Ryan Niemiec is Education Director of the VIA Institute on Character, a licensed psychologist, coach/consultant, and co-author of three books including Positive Psychology at the Movies. Ryan has a passion for teaching, practicing, and/or writing about character strengths, mindfulness, and the portrayal of positive phenomena in movies.
\end{abstract}

\section{How long have you been involved in the field of positive psychology?}

Well, that depends on your definition of positive psychology really. If you consider the field of positive psychology as starting when Martin Seligman coined the field in 1999 or so, I became interested at that point and read the original papers in the American Psychologist in the year 2000 and pushed on from there. But, if you consider positive psychology as starting long before Marty's articulation then I'd say that in the late 90s, I was infusing the principles of positive psychology into my practice as a psychologist. I always tried to embed ideas of strengths and happiness and wellbeing and take a more holistic look at people in my practice, rather than limiting myself to 'what's wrong?'.

\section{What prompted you to become interested in the area of positive psychology?}

Back in the mid to late 90s I was interested in what you might say is 'holistic health', or the biopsychosocial-spiritual model, where we look at the individual as a whole, rather than just relying on traditional training in psychology that had camps predominately focused on working with just the person's thinking or just their past experiences. I was always really interested in learning about those dimensions but going beyond that into the social realm, the cultural realm, the spiritual realm, and seeing people as so much more than just a couple of different parts, and more than the sum of their parts. So, integrating this with an inclination towards looking at what was going right with people is what primed me to be interested in this field. It was that push towards a bigger, more holistic view, that made me want to gravitate toward positive psychology and infuse that more into my work.

\section{Was there any key event that led you to stumble into positive psychology or did it just happen over time?}

It was a gradual process over time, rather than one catalyst. I've had some major events happen that have moved me forward deeper and deeper into the field of positive psychology though. For example, my connection with VIA. Prior to working at the VIA Institute on Character, I'd been involved in positive psychology for quite some time and had co-authored the book 
Positive Psychology at the Movies, which looked at character strengths portrayed in movies; and at that time, which was 2007 or so, I had not known about the existence of the VIA Institute because it was the VIA Survey and VIA Classification that were prominent at that point. I was living in St. Louis [USA] with my wife and we had decided to move closer to our families (more East). We decided to move to Cincinnati, without knowing much about the city, yet it felt right. So it was an intuitive move. This is particularly striking because we were making this decision at the worst time in the American economy since the great depression, that's in about 75 years or so, we had to buy a house, sell a house, and look for two jobs, but something told us it was the right thing to do and we were just going to do it. And while I was sitting in St. Louis one day I sent a message to a positive psychology listserv about the Positive Psychology at the Movies book and I got this query from Neal Mayerson, the chairman of VIA. My wife happened to be looking over my shoulder at the e-mail message and she noticed that the phone number in the signature line was a Cincinnati number, exactly where we were headed. This connection eventually led to VIA hiring me to help educate practitioners on how to bridge the science and practice of character strengths. So, you could call this a stumbling deeper in the thicket of positive psychology, events of synchronicity, luck, or some combination of each.

\section{In general terms what do you think are some of the distinctive features of positive psychology?}

I might be a little biased, but I, and many others, see the work on character strengths as being the backbone to positive psychology. I say that because whatever theory or approach or topic people are interested in, you can apply character strengths to it, or that particular topic stems from character strengths. For example, a lot of people are interested in happiness, or they are interested in positive emotions, and we can clearly link character strengths to those two topics. We can say that the practice of character strengths is quite strongly connected with these two areas. For example, Marty Seligman has shared how he views character strengths as the underpinnings of each area of his theory of authentic happiness, as well as his PERMA theory of wellbeing. One of the hottest topics in positive psychology is resilience. We can link in character strengths with resilience, and what character strengths an individual can use to become more resilient. Or another major area in positive psychology that is emerging for positive psychologists is mindfulness, even though mindfulness has its own following of practitioners and researchers independent of positive psychology. But when we look at the actual definition of mindfulness, researchers in studying it have formed a consensual definition, or as close to one as one can get. What it really boils down to is two character strengths: the self-regulation of our attention, and taking an attitude of curiosity and openness and acceptance. Another big area is the area of the positive institutions, which then moves into the wider areas of 'positive nations' and 'positive cultures', and creating a healthier world really. Here, we can explore how character strengths might be ingredients of a positive or virtuous institution and contribute to a healthier society. These are some of the major areas in positive psychology, and some that I am interested in myself, and we can link character strengths as underpinnings to these, as parts of the process, or as outcomes.

\section{What would you say is the big goal of positive psychology, as a field?}

There are probably several goals. But one that stands out most is the need to gain more appreciation by psychology as a whole and by other practitioners, and by the general public. I think that positive psychology has made incredible strides and has moved way beyond so many of the nay-sayers and people that were skeptical, suggesting that positive psychology 
was just a fad, and that it was going to be done and over in just a few years. They turned out to be completely wrong on that. But still, I think that positive psychology has a long way to go in terms of getting into every household, and directly impacting people of every nation, and getting deeper into government, and schools, and businesses, and every therapist's office, to be able to take more of the whole picture of the human being.

\section{How can we do this? What's the best way?}

Perhaps it's just me being impatient, but from what I can tell at IPPA [International Positive Psychology Association] and other organizations and from talking to leaders in the field it seems like we're all doing our part, and we are going in the right direction. As long as organizations like IPPA, and VIA, and other organizations can stay strong to continue to educate and promote the findings to both the general public and to various professions, then I think this will continue to grow. There are positive psychology educators, consultants and practitioners going into governments, into business, and into the school systems around the world. This is the path. We need to build and expand upon this in terms of the positive psychology principles that are taught, the professions that integrate them, and the countries this is done in. So it's coming, and it's better than any of us thought it would have been, but there's still a long way to go.

\section{What do you think is the best way that positive psychology can make this world a better place?}

A very challenging question! If each person can do their part, then that is going to spread. I think of 'pay it forward' as a good example. If each practitioner, educator, researcher, can pay this work forward, and gives positive psychology away to $\mathrm{X}$ number of people or students, and puts forth the best that they know about positive psychology to those people, maybe it's to clients, maybe it's to other colleagues, to family members, to students, and then those people can pay it forward to more individuals. We can create and spread that web of positivity, kindness, and goodness. This would make that goal more realistic, and every one of us could play the part right now and encourage and influence other people to influence others as well.

\section{What would you say is your proudest moment in the field of positive psychology over the last 10 years?}

I'm probably most proud when doing a one-on-one meeting with someone, for example a coaching type session, and applying some principles of strengths-based practice in positive psychology, and that individual completely and totally has a 180 degree shift. For example, I'm thinking of one person in particular who had been suffering tremendously over the years and who felt very weak, who had not acknowledged any of her strengths, and who had started to learn about them but still had a significant amount of blindness about her strengths. Through a conversation, just a simple little 30 minute conversation, she began to look at herself in a completely different way and began to say that she had hope now, and how she could go about making some of the tough decisions that she was facing. So that's one of the most down-toearth things that I'm proud of. I am also proud of that person in that moment for making that shift. I'm also honored to be able to stand up in front of a large group of people, for example a few-hundred people at an IPPA conference or the European Conference on Positive Psychology, and to have the opportunity to talk about something that I'm very passionate about. For example, at the beginning of an international presentation recently I showed a close- 
up image of my newborn son making eye contact with me and I spoke of how there's a moment when he rotates his head and suddenly 'sees' me. I related this to how the practitioner can shift their own perceptions and 'see' their client's core character strengths. The audience gave me an ovation for this example. I was only 5 minutes into a 2-hour talk and I was getting an ovation - and the fact that it related to my son - that was a thrilling and proud moment.

\section{What's one piece of advice for aspiring positive psychology researchers and practitioners?}

To stay true to the field. That is, to stay true to the science in terms of the research, and stay true to thinking 'how does this translate into best practice?'. That's what positive psychology is all about - linking the two.

\section{Who do you look up to in the field of positive psychology?}

I look up to a lot of people. To be honest, I actually look up to every researcher in positive psychology, and that's the truth. I don't look up more to the popular figures or those that garner the most attention. I'm a practitioner and an educator so I look up to those that have mastery in an area that I've not mastered myself, thus I appreciate researchers. I think of someone like Rhett Diessner, who works at a small university in the state of Idaho. I look up to him. He's doing research with some of his students on 'appreciation of beauty'. I also look up to some of the larger scale researchers like Chris Peterson, who has done so much wonderful work in the field.

What do you think fits well along with strengths, so to speak? For example if you were going to work with someone on strengths, what would you additionally work with them on?

I would link in mindfulness, as that's what I'm also passionate about as a practitioner. I am beginning to study this interrelationship more closely. But I would weave this in, helping the individual to become more mindful of those strongest qualities inside them and the resources around them, and using mindfulness and strengths to deal with problems and with different issues; and how we can be mindful of overusing our character strengths or not using enough of them. I would link how to find that balance and how to be mindful of applying strengths in the right context and how to be mindful of how to use strengths in order to flourish. I think that they are natural bedfellows. I recently created and piloted a new eight-week programme focusing on the integration of mindfulness and character strengths, and I will look forward to advancing this and sharing it in the future.

\section{Is there anything else you'd like to add that I haven't asked about that you think would be useful for a person looking at moving into the world of positive psychology?}

I see movies as an incredible medium for helping people to realize their potential, to become better, and to apply positive psychology principles. One of the major reasons for me saying this is that when an individual takes an approach to movies, whether they are paying attention to themselves, or paying attention to the impact of the character on the screen, they will often feel inspired or feel what researcher Jonathan Haidt has coined as 'elevation'. Movies can be cinematically elevating in that we observe a character doing something inspiring, or we observe a character displaying bravery or wisdom or perseverance in some strong way, and maybe we need to build that strength within ourselves, or maybe it just reflects a part of ourselves. We feel the physiological sensations of tingling and warming in the chest; and then, most importantly, we are motivated to do good, or are motivated to be better people, or are 
motivated to be more altruistic. This is an exciting area that needs to have more research. It also links in with the question about making the world a better place, because movies are something that can transcend cultures and nations and therefore they have potential to reach each person and can help people to be stronger and to flourish.

\section{Author}

Aaron Jarden

Open Polytechnic of New Zealand

aaron.jarden@openpolytechnic.ac.nz 